\title{
APLICAÇÕES DA QUÍMICA COMBINATÓRIA NO DESENVOLVIMENTO DE FÁRMACOS
}

\author{
Ricardo L. Araújo Dias e Arlene G. Corrêa* \\ Departamento de Química, Universidade Federal de São Carlos, CP 676, 13565-905 São Carlos - SP
}

Recebido em 17/2/00; aceito em 28/8/00

\begin{abstract}
APPLICATIONS OF COMBINATORIAL CHEMISTRY TO DRUG DEVELOPMENT. Combinatorial Chemistry has become a very efficient methodology in drug research. Recent progress in combinatorial synthesis performed both in solid and solution phase have led to a change in the paradigm for the identification and optimization of lead compounds. This article gives an overview of the principal characteristics of combinatorial libraries and some examples of the application of this methodology in the identification of test compounds and lead compound optimization, either from synthetic or natural sources.
\end{abstract}

Keywords: combinatorial chemistry; drug development; small molecule libraries.

\section{INTRODUÇÃO}

O processo de desenvolvimento de fármacos incorporou profundas transformações no decorrer da última década. Com o avanço da Biologia Molecular inúmeros processos biológicos foram identificados, fornecendo assim diversos novos alvos macromoleculares passíveis de intervenção terapêutica. Paralelamente, a miniaturização e o desenvolvimento de dispositivos automatizados para a realização de ensaios biológicos automatizados (do inglês, High Throughput Screening, HTS) possibilitaram a avaliação de uma grande quantidade de substâncias frente a estes novos alvos. A resposta a esta demanda crescente por substâncias estruturalmente inovadoras para avaliação farmacológica estabeleceu um novo paradigma na busca por compostos-protótipo e na otimização/desenvolvimento dos já existentes. A preparação conjunta - na forma de misturas - ou paralela - na forma de substâncias puras - de coleções com grande número de compostos estruturalmente relacionados, com sequências de reação semelhantes e com diversidade estrutural gerada pela introdução de blocos de construção com diferentes grupos substituintes promoveu uma grande transformação em relação aos métodos até então utilizados no planejamento de fármacos ${ }^{1-5}$.

Ainda incipiente e restrita à preparação de coleções de peptídeos e nucleotídeos, a Química Combinatória apresentou um crescimento vertiginoso desde a preparação de uma coleção combinatória de benzodiazepinas em 1992, trabalho pioneiro realizado por Ellmann e col., que consistiu na primeira coleção combinatória de moléculas orgânicas pequenas ${ }^{6}$. A Química Combinatória extrapolou a esfera da Química Medicinal e vem sendo crescentemente utilizada nas áreas de agroquímicos, catálise homogênea e heterogênea, biocatálise, novos materiais, entre outros ${ }^{1-4}$. A Figura 1 apresenta a evolução do número de publicações científicas na área de Química Combinatória ao longo dos últimos anos ${ }^{7}$.

As coleções combinatórias podem ser divididas entre as preparadas por síntese paralela, onde as etapas sintéticas são realizadas simultaneamente, mas cada composto é preparado em um compartimento de reação separadamente, e as preparadas na forma de mistura, ou seja, a um substrato é adicionado uma mistura de reagentes (building blocks), fornecendo então uma mistura de produtos. Por demandar um ensaio farmacológico por composto preparado, a síntese paralela é empregada principalmente na preparação de coleções combinatórias menores (com até algumas centenas de compostos). Já as coleções com-

e-mail: agcorrea@dq.ufscar.br

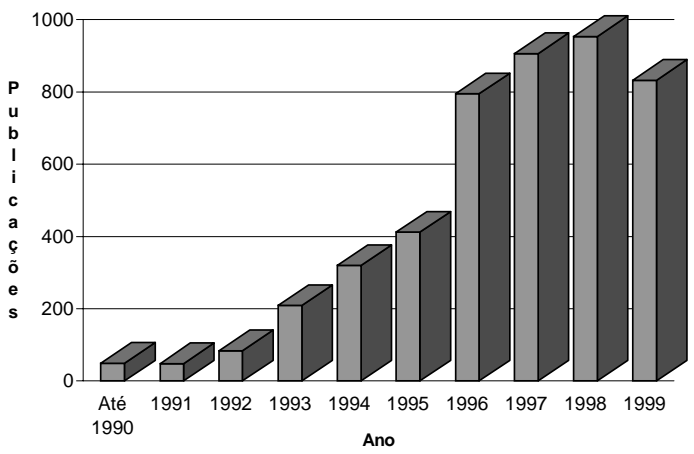

Figura 1. Crescimento do número de publicações científicas relacionadas com Química Combinatória.

binatórias preparadas na forma de mistura possuem, em alguns casos, milhões de compostos. $\mathrm{O}$ procedimento utilizado para a identificação dos compostos ativos numa coleção deste porte depende, basicamente, da estratégia previamente escolhida para a construção da coleção. Existem alguns métodos para esta identificação do composto ativo na mistura, ou desconvolução da coleção ${ }^{8-10}$, sendo que a estratégia a ser utilizada está diretamente ligada ao protocolo pelo qual a coleção combinatória foi preparada. Dentre as várias formas de se realizar esta identificação pode-se citar o fracionamento por cromatografia líquida (CLAE) orientado pelos ensaios farmacológicos; a análise passo a passo dos componentes de diversidade introduzidos (método "mistura-divide"); a preparação de coleções codificadas com "etiquetas moleculares"; até o uso de dispositivos mais sofisticados como microchips que "acompanham" toda a rota sintética e gravam, por sinais de radiofrequência recebidos, as condições de reação utilizadas ${ }^{8-10}$.

As coleções combinatórias podem ainda ser divididas entre aquelas preparadas em solução, assim como na síntese orgânica clássica; e aquelas onde a síntese dos componentes é realizada em fase sólida, ou seja, os compostos são preparados "ligados" a uma resina polimérica, de onde são posteriormente clivados.

\section{SÍNTESE EM FASE SÓLIDA}

A síntese em fase sólida apresenta como maior vantagem a simplicidade para purificação dos produtos obtidos. A filtração, seguida de sucessivas lavagens do suporte polimérico com diferentes solventes são suficientes para se atingir a pureza necessária dos produtos. Isto permitiu então que diversas metodologias classicamente empregadas em síntese orgânica 
pudessem ser adaptadas para a síntese em fase sólida, resultando inclusive na automação de vários destes processos. Como principais desvantagens desta estratégia pode-se destacar a necessidade de se utilizar grandes excessos dos reagentes a fim de se assegurar a completa conversão do substrato, uma vez que a difusão dos reagentes pela matriz polimérica é um fator limitante; a escassez de métodos analíticos para o monitoramento das reações; e a necessidade de se adaptar as metodologias sintéticas já conhecidas para a utilização em fase sólida ${ }^{1,2}$. Nos últimos anos muitos grupos de pesquisa têm se dedicado a contornar estes problemas, através do desenvolvimento de métodos analíticos para o monitoramento de reações em fase sóli$\mathrm{da}^{11}$, pela geração de uma enorme variedade de estruturas químicas e grupos funcionais ligados ao suporte sólido, comportando-se como conectores químicos (do inglês, linkers) ${ }^{12}$ e por inúmeros trabalhos para adaptação de metodologias sintéticas, o que resultou em grande crescimento no número de metodologias sintéticas já adaptadas para síntese em fase sólida ${ }^{13-16}$.

A coleção combinatória pioneira de 1,4-benzodiazepinas $\mathbf{1}$, preparada por Ellman e col. ${ }^{6}$, foi obtida por síntese paralela, a partir da ligação de duas diferentes aminobenzofenonas 2 ao suporte polimérico através de um conector químico sensível a meio ácido, após proteção do grupo amino com a introdução do grupo FMOC, gerando a aminoacetona 3 (Esquema 1). A posterior desproteção do grupo amino, seguida de acilação com diferentes fluoretos de aminoácidos FMOC-protegidos forneceu a amida 4 (onde $\mathrm{R}^{3}$ corresponde à cadeia lateral), introduzindo um novo fator de diversidade. A remoção do grupo protetor FMOC e consequente ciclização levou aos derivados benzodiazepínicos 5 . Em seguida foi realizada a $\mathrm{N}$-alquilação com diferentes agentes alquilantes, fornecendo a benzodiazepinona 6, com a introdução de mais um fator de diversidade $\left(\mathrm{R}^{4}\right)$. Por fim, foi feita a clivagem do suporte polimérico, resultando então na obtenção das 1,4-benzodiazepinas 1 em solução.
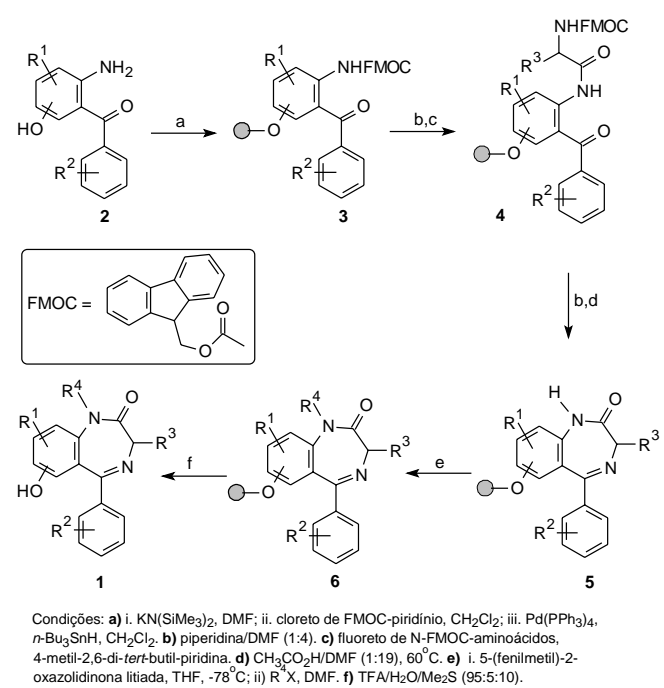

Esquema 1.

\section{SÍNTESE EM SOLUÇÃO}

A preparação de coleções combinatórias em solução tem como principal vantagem utilizar-se das mesmas condições de reação empregadas na síntese orgânica clássica, sem a necessidade de utilização dos excessos de reagentes requeridos pela síntese em fase sólida. A principal desvantagem da preparação de coleções combinatórias em solução com grau de pureza confiável para posterior avaliação farmacológica consiste na complexidade de purificação dos produtos. Sem a utilização de métodos simples e eficientes para a purificação dos produtos, a química combinatória em solução ficou inicialmente restrita à sequências de reação curtas ${ }^{17}$. Com isto, estratégias vêm sendo aplicadas para superar esta limitação de forma a permitir a preparação de moléculas que requerem rotas mais longas e complexas. Neste sentido, pode-se destacar a utilização de reagentes e catalisadores imobilizados em suportes sólidos e também a utilização de resina polimérica para remoção de excesso do reagente e/ou substrato não convertido (scavenger) (Tabela 1) ${ }^{17-18}$.

Tabela 1. Alguns exemplos de resinas empregadas na preparação de coleções combinatórias em solução. ${ }^{17-18}$

\begin{tabular}{ll}
\hline Reagente Imobilizado & Uso \\
\hline Poliestireno-EDC & Reagente de acoplamento \\
Amberlite ${ }^{\circledR}$ IRA-400 borohidreto & Agente redutor \\
Poliestireno-aminometil & Scavenger \\
Poliestireno-poliamina & Scavenger \\
Poliestireno-isocianato & Scavenger \\
Poliestireno-aldeído & Scavenger \\
Poliestireno-morfolina & Base, Scavenger \\
Resinas de troca iônica ácidas & Ácido/base, Scavenger \\
\hline
\end{tabular}

Kaldor e col. relataram uma estratégia utilizada na preparação de uma coleção de uréias em solução, onde os isocianatos residuais foram removidos das misturas de reação pela adição de uma resina poliestireno-aminometil (Esquema 2,a) ${ }^{19}$. A purificação das misturas se faz pela simples filtração das mesmas quando não mais se detecta a presença de isocianatos em solução. Esta mesma estratégia foi utilizada para a remoção do excesso de aminas nas misturas de reação de uma coleção de b-aminoálcoois (Esquema 2,b) ${ }^{20}$. Booth e Hodges relataram a preparação de uma coleção de derivados pirazólicos a partir da condensação de uma 1,3-dicetona com diferentes ácidos 4fenilhidrazina carboxílicos catalisada por morfolina imobiliza$\mathrm{da}^{21}$. O excesso do ácido foi então removido com uma resina poliestireno-isocianato, e os produtos foram obtidos em $97 \%$ de pureza após filtração e remoção do solvente (Esquema 2,c).

$$
\text { filtração } \mathrm{R}^{1} \mathrm{R}^{2} \mathrm{NH} \frac{\mathrm{R}^{3} \mathrm{NCO}(1,25-2 \text { eq.) }}{\mathrm{CHCl}_{3} \text {, t.a. }}
$$
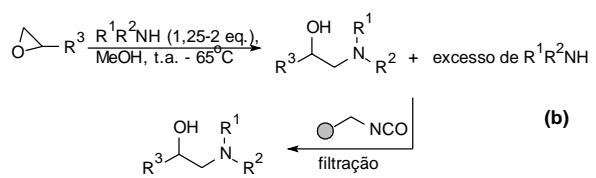

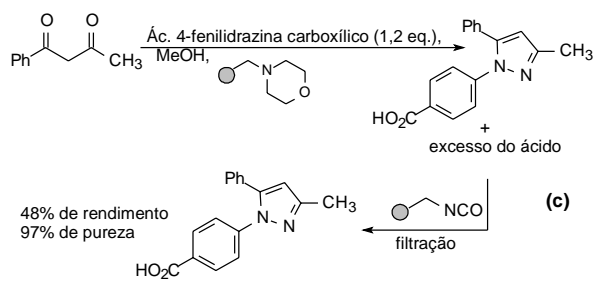

Esquema 2.

\section{SÍNTESE EM POLÍMEROS SOLÚVEIS}

Uma estratégia muito elegante foi relatada por Janda e col., que consistiu na utilização de um polímero solúvel para a preparação de coleções combinatórias ${ }^{22}$. O homopolímero mais 
comumente empregado para imobilização do substrato é o polietilenoglicol, principalmente devido à sua alta solubilidade frente a uma grande variedade de solventes orgânicos ${ }^{22}$. A reação pode então ser realizada na forma homogênea sem a necessidade de nenhuma adaptação experimental. Ao término da mesma, após a adição de um solvente apropriado, o homopolímero pode ser seletivamente precipitado na forma de um sólido cristalino. Este sólido, obtido após filtração, lavagem e secagem pode então novamente ser submetido à próxima etapa sintética ou à clivagem, fornecendo os compostos desejados em alto grau de pureza. Esta estratégia, também denominada "síntese em fase líquida", se mostra bastante promissora, já que associa vantagens da síntese em fase sólida, como a simplicidade na purificação dos produtos, e da síntese em solução, dispensando o uso de excesso de reagentes e de adaptação de metodologias sintéticas. Além disto, os polímeros solúveis custam entre 10 e 500 vezes menos que as resinas poliméricas comercialmente disponíveis para síntese em fase sólida ${ }^{23}$.

\section{QUÍMICA COMBINATÓRIA APLICADA AO DESCOBRIMENTO DE FÁRMACOS}

O processo de descobrimento de um novo fármaco pode ser feito através de várias estratégias, todas envolvendo diversas etapas. Uma destas estratégias parte da escolha de um alvo macromolecular, seguida pela validação deste alvo e do ensaio bioquímico/farmacológico, passando pela identificação de um composto-protótipo, modificação/otimização deste compostoprotótipo até a aprovação nos testes clínicos em humanos. O tempo total envolvido em todo o processo é de cerca de 15 anos, sendo que aproximadamente um terço deste tempo corresponde à identificação e otimização do composto-protótipo. ${ }^{24} \mathrm{Em}$ decorrência do aumento significativo no prazo de avaliação clínica de candidatos a drogas ao longo das duas últimas décadas (Figura 2), os grupos envolvidos com estas pesquisas (particularmente as grandes indústrias farmacêuticas) atuam sistematicamente visando a diminuição do tempo de desenvolvimento e otimização da substância candidata à fármaco.

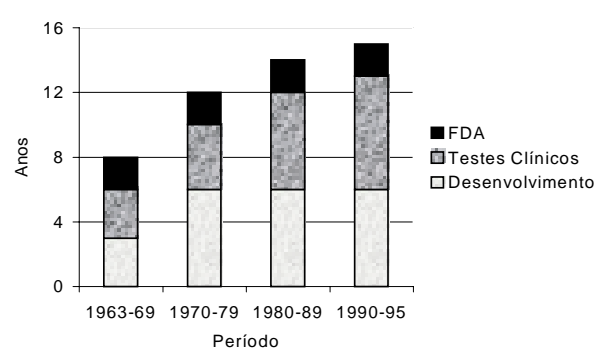

Figura 2. Componentes do prazo total de desenvolvimento de uma nova droga nos Estados Unidos. FDA (Food and Drug Administration) é a agência norteamericana de regulamentação e controle do setor de medicamentos ${ }^{24}$.

A geração de grandes coleções de moléculas orgânicas estruturalmente diversas e a avaliação farmacológica sistemática destas possibilita a identificação de ligantes potentes e seletivos em intervalos de tempo menores. Além disso, a preparação de coleções combinatórias de derivados com estrutura básica comum a fármacos já conhecidos assegura grande possibilidade de o composto-protótipo apresentar bons resultados em etapas cruciais do processo, onde são avaliadas propriedades como lipossolubilidade, biodisponibilidade, toxicidade, entre outras 2,7 . Estas coleções de moléculas com estruturas "privilegiadas" são muito encontradas na literatura, sendo que as coleções de benzodiazepinas são um bom exemplo desta estratégia ${ }^{6}$.

Embora inicialmente algumas empresas tenham comercializado coleções combinatórias contendo milhares de compostos, planejadas para cobrir ampla faixa de diversidade estrutural e para ser utilizadas na etapa de identificação do composto-protótipo, a maior parte das pesquisas atuais está voltada para a preparação de coleções menores, mais específicas, visando a otimização de compostos-protótipo. Neste sentido, reafirma-se o papel desempenhado pelos produtos naturais como uma importante fonte para identificação de compostos-protótipo. Nos últimos anos, é crescente o número de coleções combinatórias de derivados de produtos naturais bioativos escolhidos como composto-protótipo ${ }^{25}$.

As próximas seções deste artigo ilustrarão estratégias combinatórias aplicadas na identificação de compostos-protótipo, na otimização de compostos-protótipo e coleções combinatórias baseadas em produtos naturais.

\section{IDENTIFICAÇÃO DE UM COMPOSTO-PROTÓTIPO}

Coleções combinatórias preparadas com o intuito de se identificar um composto-protótipo, em geral, possuem milhares de substâncias que são testadas sob a forma de misturas de componentes. Algumas destas coleções são preparadas a partir da escolha de um composto polifuncionalizado (core), que dá origem a uma série de outros após reações com um conjunto de compostos estruturalmente diversos mas com a mesma funcionalidade/reatividade. A vantagem desta estratégia é que, em virtude da grande reatividade deste composto-base, o consumo total dos reagentes é assegurado, de forma que a pureza da coleção permite sua utilização direta nos ensaios farmacológicos. A coleção combinatória de tetraamidas preparada por Rebek, Jr. e col. consistiu na reação do cloreto de acila tricíclico 7 com 19 aminoácidos o que, teoricamente, forneceria 65341 tetraamidas a ser testadas como inibidores da enzima tripsina (Esquema 3$)^{26}$. A avaliação farmacológica dos componentes da coleção levou à identificação do composto 8, que apresentou inibição da enzima tripsina $\left(K_{i}=9 \pm 2 \mu \mathrm{M}\right)$.

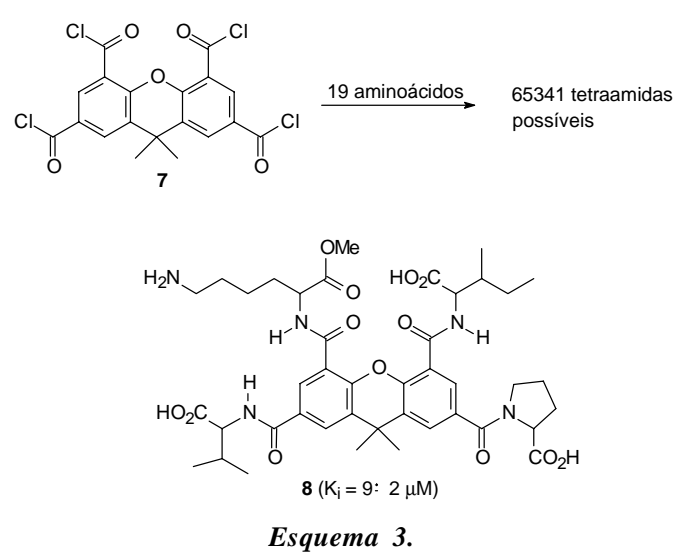

Powers e col. reportaram a preparação de coleções combinatórias derivadas da coleção de 1280 chalconas de estrutura geral 9 previamente preparadas (Esquema 4$)^{27}$. A primeira coleção foi obtida a partir da condensação aldólica de 32 acetofenonas e 40 aldeídos aromáticos, fornecendo as 1280 chalconas 9. De posse desta coleção, foram preparadas outras 9 sub-coleções a partir de 9 sequências de reação realizadas de forma automatizada, em paralelo, resultando então num total de 74.000 compostos. Como exemplo pode-se citar a reação da coleção 9 com cloridrato de hidroxilamina que forneceu 1280 compostos heteroaromáticos contendo o anel isoxazolínico 10. As reações com 6 diferentes fenilidrazinas, 6 diferentes acetoacetanilidas e 6 diferentes aminobenzoimidazóis forneceram, respectivamente, as sub-coleções 11, 12 e 13, contendo cada uma 7680 compostos (Esquema 4). Os 74.000 compostos preparados foram comparados estruturalmente com uma base de dados contendo 87.500 substâncias farmacologicamente 
importantes. A similaridade estrutural de alguns dos compostos preparados com algumas das substâncias constantes na base de dados é que orientou os ensaios farmacológicos pelos quais as coleções combinatórias foram avaliadas.
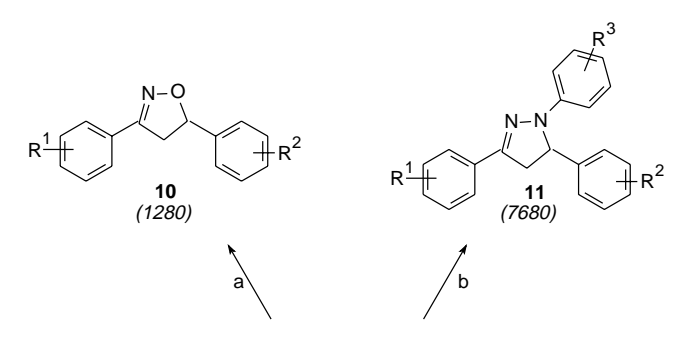<smiles>C[13CH]1CCCCC1</smiles><smiles></smiles>
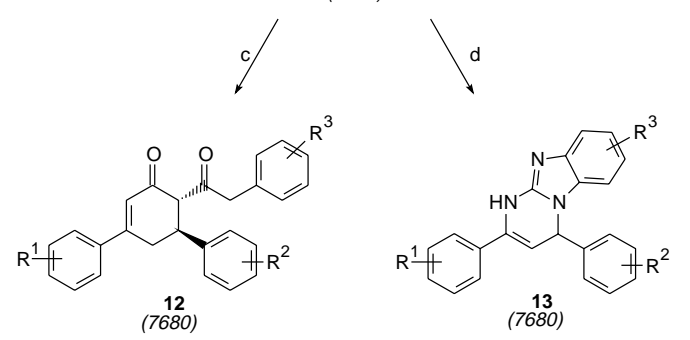

a) $\mathrm{HONH}_{2} . \mathrm{HCl} / \mathrm{EtOH}, \mathrm{NaOH}$ (1eq.). b) 6 Fenilidrazinas, $\mathrm{NaOH}$ (1eq.), EtOH, $70^{\circ} \mathrm{C}$. c) 6 Acetoacetanilidas, $\mathrm{NaOH}$ (1eq.), $\mathrm{EtOH} / \mathrm{H}_{2} \mathrm{O} 4: 1,70^{\circ} \mathrm{C}$. d) 6 Aminobenzoimidazóis, $\mathrm{NaOH}$ (1eq.), $\mathrm{EtOH} / \mathrm{H}_{2} \mathrm{O} 4: 1,70^{\circ} \mathrm{C}$

\section{Esquema 4.}

Conforme mencionado anteriormente, uma das estratégias que vem sendo empregada por alguns grupos de pesquisa consiste em se preparar coleções combinatórias das estruturas chamadas "privilegiadas". Esta classificação foi direcionada a algumas classes de compostos que apresentam amplo espectro de atividades biológicas, além de propriedades farmacológicas apropriadas para uso terapêutico. Um exemplo desta classe de estruturas são as benzodiazepinas, que apresentam atividade biológica frente a diversos alvos biológicos importantes. $\mathrm{O}$ sedativo Valium ${ }^{\circledR}$ (Hoffman-La Roche) é um exemplo de uma benzodiazepina em uso terapêutico.

Posteriormente à primeira coleção de benzodiazepínicos (Esquema 1) ${ }^{6}$, Ellman e col. prepararam, com algumas modificações na rota sintética, uma nova coleção de 1,4-benzodiazepinas (Esquema 5$)^{28}$. A aminoestanana 15 foi preparada em 5 etapas a partir de $p$-aminofenol 14. O acoplamento de Stille com 20 diferentes cloretos de ácidos comerciais $\left(\mathrm{R}^{1} \mathrm{COCl}\right)$ e posterior desproteção do grupo amino forneceu a aminocetona 16. Após a conclusão da síntese através do mesmo procedimento descrito anteriormente (Esquema 1), utilizando-se 35 aminoácidos e 16 agentes alquilantes, obtiveram-se as benzodiazepinas 17, num total de 11.200 diferentes compostos.

As prostaglandinas constituem uma outra importante classe de estruturas "privilegiadas". Estas moléculas desempenham importante papel fisiológico, estando diretamente envolvidas em processos inflamatórios, regeneração de tecidos e resposta imunológica. ${ }^{29}$ Devido à pronunciada biodisponibilidade desta classe de compostos, diversos derivados sintéticos baseados no seu esqueleto carbônico são preparados para os mais diferentes alvos biológicos. Janda e col. relataram uma estratégia para preparação de derivados de prostaglandinas $\mathrm{E}_{2} \mathbf{1 8}\left(\mathrm{PGE}_{2}\right)$ utilizando-se os blocos de construção 19-21 para a síntese em fase líquida (Esquema 6$)^{29}$. O polímero solúvel escolhido foi o poliestireno clorometilado não entrecruzado $\mathbf{2 2}$, tendo sido modificado para o conector químico derivado do DHP 23 para imobilização de álcoois. A ligação do composto 19 ao suporte foi realizada por catálise ácida (PPTS, para-toluenossulfonato de piridínio), fornecendo o éter $\mathbf{2 4}$. A adição do cuprato de $\mathbf{2 0}$ seguido de captura do enolato por TMSCl forneceu o silienol éter 25. A alquilação de $\mathbf{2 5}$ utilizando o triflato $\mathbf{2 1}$ levou ao composto 26, que após hidrogenação catalítica $\left(\mathrm{H}_{2}, \mathrm{Pd}_{-} \mathrm{BaSO}_{4}\right)$ forneceu a prostaglandina imobilizada 27. A clivagem do polímero com HF aquoso forneceu a estrutura-alvo 18, em 8 etapas e $37 \%$ de rendimento global ${ }^{29}$.

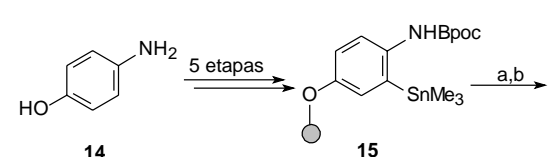<smiles>[R]C1=NC(=C)C([R7])N([Tl])c2ccc(O)cc21</smiles>

Condiçōes: a) Pd (0), $\mathrm{R}^{1} \mathrm{COCl}$. b) $\mathrm{TFA} / \mathrm{CH}_{2} \mathrm{Cl}_{2}(3: 97)$.

Esquema 5.
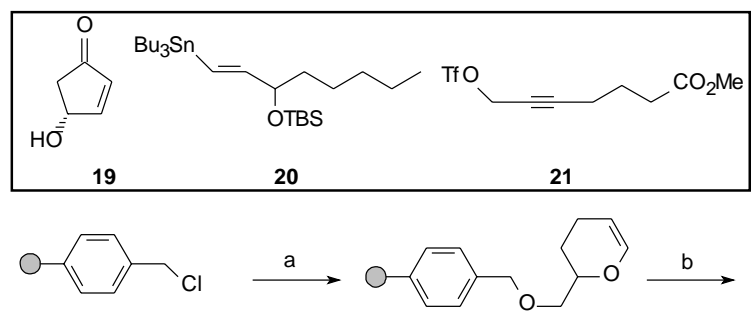

22

23<smiles>C#C[C@H](O)O[C@H]1C=CC(=O)C1</smiles>

24
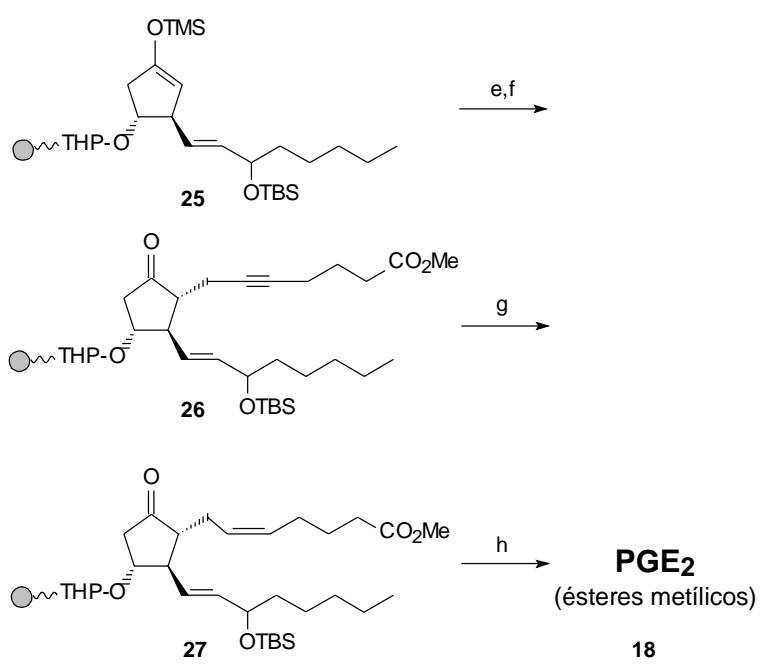

Condições: a) 6-(hidroximetil)-DHP (3 eq), $\mathrm{NaH}$ (3,3 eq.) DMA, t.a., 24h. b) 19 (3 eq.), PPTS ( 0,5 eq.), $\mathrm{CH}_{2} \mathrm{Cl}_{2}, 40^{\circ} \mathrm{C}, 16$ h. c) 20 (4,2 eq.), LiCuCNMe 2 ( 3,9 eq.), THF, $-78^{\circ} \mathrm{C}$, 15 min. d) i. TMSCl (15 eq.), $-78^{\circ} \mathrm{C}, 30 \mathrm{~min}$; ii. TEA (30 eq.), $0^{\circ} \mathrm{C}, 15 \mathrm{~min}$. e) MeLi (3 eq.), THF, $-23^{\circ} \mathrm{C}, 20 \mathrm{~min}$ f) i. 21 ( 6 eq.) $,-78^{\circ} \mathrm{C}, 10 \mathrm{~min}$; ii. $-23^{\circ} \mathrm{C}, 30 \mathrm{~min}$. g) $\mathrm{H}_{2}$ $5 \%{\mathrm{Pd}-\mathrm{BaSO}_{4} \text {, quinolina, } \mathrm{PhH} / \text { ciclohexano (1:1), } 48 \text { h. h) HF aquoso } 48 \% / \mathrm{THF}}$ $5 \% \mathrm{Pd}-\mathrm{BaSO}_{4}$, quinolin,
$(3: 20, \mathrm{v} / \mathrm{v}), 45^{\circ} \mathrm{C}, 6 \mathrm{~h}$.

Esquema 6. 


\section{OTIMIZAÇÃO DE UM COMPOSTO-PROTÓTIPO}

A identificação de um inibidor com boa biodisponibilidade é um desafio muito maior do que a de um ligante de excepcional afinidade in vitro. Por isto, muitas substâncias eleitas como protótipos, por apresentarem bons resultados em ensaios in vitro, não avançam no processo de desenvolvimento por não apresentarem atividade in vivo correspondente. A preparação de coleções combinatórias de derivados do composto-protótipo pode auxiliar na busca por um ligante com propriedades farmacocinéticas apropriadas e também com maior afinidade pela macromolécula-alvo. Muito comumente tem-se relatado na literatura a preparação destas coleções como ferramenta para a otimização do composto-protótipo (lead optimization), que pode ser tanto um produto natural, um composto proposto por modelagem molecular, quanto um composto sintético, identificado numa primeira coleção combinatória.

Um bom exemplo desta estratégia foi reportado por Hoekstra e col. com a preparação de uma coleção de derivados da nipecotamida $\mathbf{2 8}$, um inibidor da agregação plaquetária induzida por colágeno ${ }^{30}$. A utilização desta substância na terapia antitrombótica é inviabilizada por sua reduzida potência in vivo. Inicialmente foi preparada uma pequena coleção de derivados da nipecotamida 28 para avaliação do efeito de substituições bioisostéricas nas posições X e Y (Esquema 7). Posteriomente, após escolhidos os substituintes mais apropriados para as posições $\mathrm{X}$ e $\mathrm{Y}$, foram preparados derivados que avaliaram a influência do número de carbonos no anel central $(\mathrm{n}=0,1 \mathrm{e}$ 2), bem como de diferentes substituintes na posição $Z$ a partir da incorporação de $\beta$ aminoésteres 3-substituídos. Este estudo resultou na identificação do derivado 29 que, após síntese estereosseletiva, foi submetido aos ensaios farmacológicos e se mostrou 60 vezes mais potente no ensaio in vitro que 28 e cerca de 3,3 vezes mais potente no ensaio in vivo ${ }^{30}$.
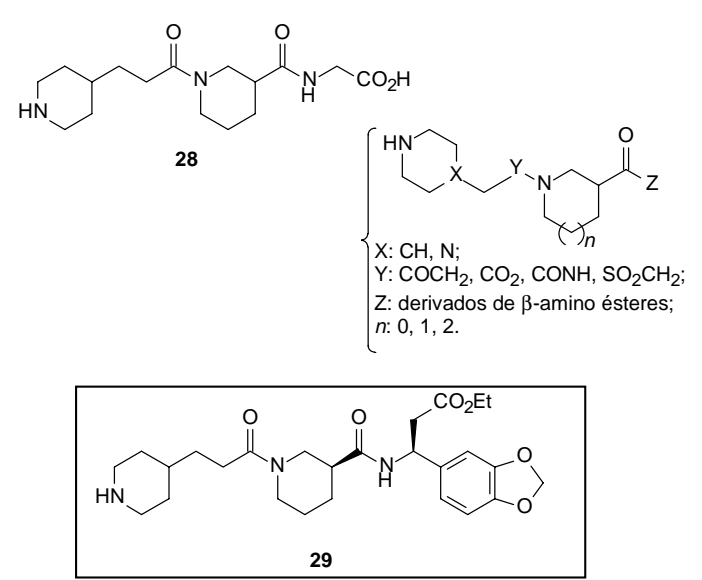

Esquema 7.

Um outro exemplo de otimização de um composto-protótipo pela preparação combinatória de derivados foi reportada por Gayo e col. que prepararam uma coleção de derivados do composto 30 (Esquema 8), inibidor dos receptores NF- $\mathrm{KB}$ e AP-1, envolvidos na ativação da transcrição, processo relacionado ao reconhecimento celular e associado a diversas respostas patológicas.17,18 A preparação da coleção combinatória em solução foi realizada pela reação de 160 diferentes aminas comerciais 31 com diferentes cloretos de ácidos heteroaromáticos 32. A avaliação dos 4.500 compostos derivados 33 resultou na identificação de potentes análogos, um deles com $\mathrm{IC}_{50}$ entre 50 e $100 \mathrm{nM}$, ou seja, entre 5 a 10 vezes mais ativos que o protótipo 30, tendo inclusive apresentado atividade em ensaios com animais ${ }^{17,18}$.

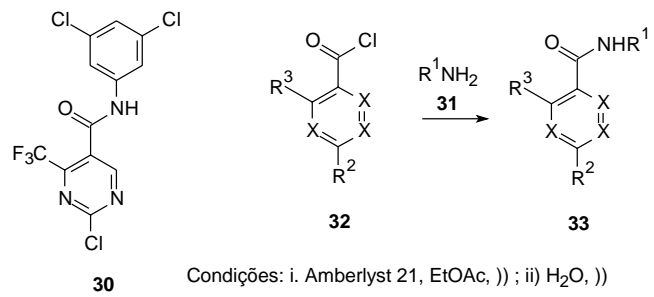

Esquema 8.

A utilização de metodologias combinatórias para a identificação de ligantes de alta afinidade no planejamento de drogas baseado na estrutura molecular da macromolécula-alvo também tem sido reportada. A identificação do composto-protótipo pode ser realizada por screenings computacionais de banco de dados de moléculas pequenas (docking de "coleções virtuais"), ou pela sugestão de um ligante a partir da análise da topografia do sítioalvo na macromolécula. Analisada a topografia do sítio-alvo, pode-se planejar os grupos farmacofóricos importantes no ligante, bem como determinar o esqueleto carbônico apropriado para a "sustentação" dos grupos farmacofóricos nas coordenadas tridimensionais ideais. A partir então da proposição, por modelagem molecular, do composto-protótipo, coleções combinatórias pequenas e sítio-dirigidas são preparadas para otimização da afinidade do ligante. ${ }^{31-33}$ Cabe aqui ressaltar ainda que coleções virtuais podem também ser estabelecidas mesmo quando não se conhece o alvo macromolecular ${ }^{34}$.

\section{COLEÇÕES COMBINATÓRIAS DE DERIVADOS DE PRODUTOS NATURAIS}

Do ponto de vista histórico, os produtos naturais sempre desempenharam um papel muito importante no processo de desenvolvimento de fármacos. ${ }^{35} \mathrm{~A}$ maior parte das drogas comercializadas é de origem natural ou produtos sintéticos inspirados em produtos naturais. Tanto os métodos clássicos de isolamento de produtos naturais quanto a utilização da tecnologia dos ensaios biológicos automatizados (HTS) na avaliação destas substâncias são uma fonte promissora de novos compostos-protótipos ${ }^{36}$.

A associação da pesquisa de produtos naturais para identificação do composto-protótipo com a preparação de coleções combinatórias para sua otimização ainda é bastante incipiente, mas tem sido crescentemente explorada. A diversidade estrutural dos produtos naturais somada à capacidade da Química Combinatória de fornecer grande número de derivados em curto intervalo de tempo sugerem uma ação sinergista na busca por novos fármacos.

As coleções combinatórias de derivados de produtos naturais encontradas na literatura distinguem-se tanto com relação à complexidade das estruturas quanto nas finalidades farmacológicas, envolvendo a preparação de derivados relativamente simples, como os da kramerixina $(\mathbf{3 4})^{37}$, do balanolol $(\mathbf{3 5})^{38}$ e da indolactama V $(\mathbf{3 6})^{39}$, até derivados mais complexos como os análogos das epotilonas A $(\mathbf{3 7})$ e B $(\mathbf{3 8})^{40}$ e das sarcodictinas A (39) e B (40) (Figura 3$)^{41}$.

As sarcodictinas A (39) e B (40) foram isoladas de corais da espécie Sarcodictyion roseum e possuem potente ação antitumoral, apresentando modo de ação semelhante ao do taxol (polimerização da tubulina e estabilização dos microtúbulos) ${ }^{42}$. A preparação da coleção combinatória de análogos das sarcodictinas empregou o intermediário avançado 41 e utilizou as posições indicadas em $\mathbf{4 2}$ para a geração de diversos derivados (Esquema 9). Coleções combinatórias de derivados estruturalmente complexos como este visam não somente a identificação de compostos mais potentes, mas também estabelecer algumas relações estrutura-atividade, de forma a identificar os grupos farmacofóricos do composto-protótipo e permitir uma 
eventual simplificação estrutural. A coleção de derivados das sarcodictinas 39-40 permitiu o estabelecimento de algumas destas relações a partir da avaliação farmacológica da mesma, tendo também resultado na identificação de alguns compostos ainda mais potentes que os produtos naturais (Figura 4).
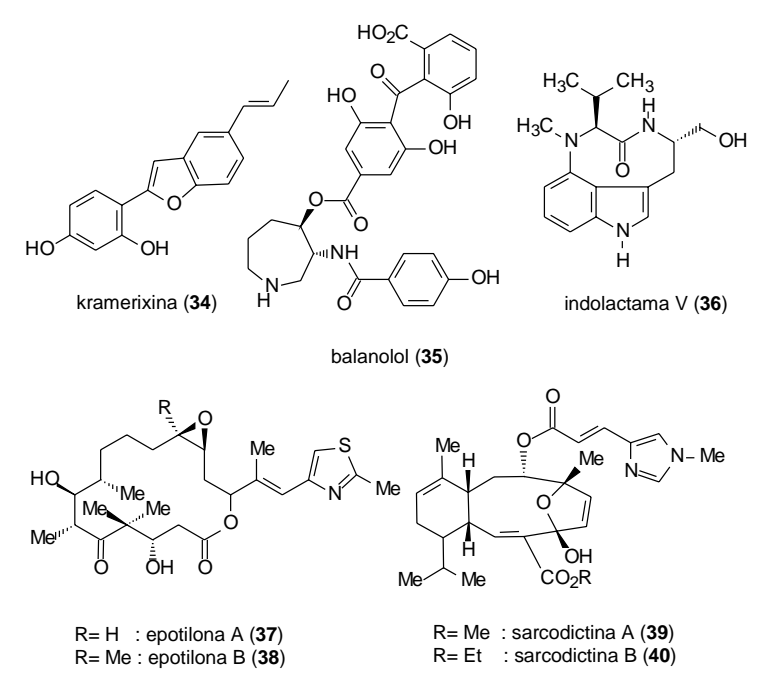

Figura 3. Alguns produtos naturais utilizados como protótipos para preparação de coleções combinatórias.

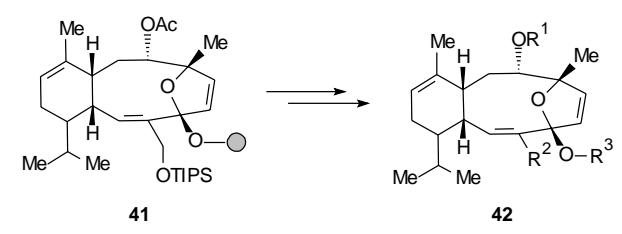

Esquema 9.

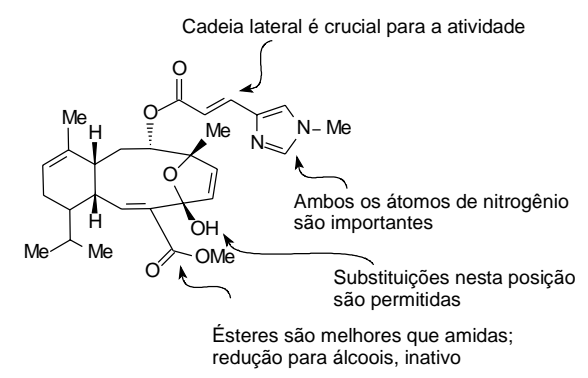

Figura 4. Algumas relações estrutura-atividade observadas para a sarcodictina $A$ (39)

\section{CONCLUSÕES}

Na última década, com os avanços na Biologia Molecular e com a automação dos ensaios biológicos, a Química Combinatória teve um grande crescimento e tem se mostrado uma poderosa ferramenta nas pesquisas de fármacos, agroquímicos, novos materiais, catálise, entre outros.

Na busca por novos fármacos, a preparação de coleções combinatórias tem auxiliado tanto na identificação de compostos-protótipo quanto na otimização destes. A preparação de coleções de derivados, cujo padrão molecular já é encontrado em moléculas endógenas ou mesmo em fármacos comerciais, é de grande utilidade para a identificação de moléculas ativas, biodisponíveis frente a vários diferentes alvos biológicos. Mais recentemente, a extensão da abordagem combinatória aos produtos naturais bioativos vem sendo realizada, também de forma bastante promissora.

\section{AGRADECIMENTOS}

Os autores agradecem à Fundação de Amparo à Pesquisa do Estado de São Paulo (FAPESP) pela bolsa de doutorado de R. L. A. D. e ao Prof. Marcus Mandolesi Sá (UFSC) pela leitura do manuscrito.

\section{REFERÊNCIAS}

1. Balkenhohl, F.; Bussche-Hünnefeld, C.; Lansky, A.; Zechel, C.; Angew. Chem. Int. Ed. Engl. 1996, 35, 2288.

2. Thompson, L. A.; Ellman, J. A.; Chem. Rev. 1996, 96, 555.

3. Ellman, J. A.; Acc. Chem. Res. 1996, 29, 132.

4. Gordon, E. M.; Gallop, M. A.; Patel, D. V.; Acc. Chem. Res. 1996, 29, 144.

5. Furlán, R. L. E.; Labadie, G. R.; Pellegrinet, S. C.; Ponzo, V. L.; Quim. Nova 1996, 19, 411.

6. Bunin, B. A.; Ellman, J. A.; J. Am. Chem. Soc. 1992, 114, 10997.

7. Lebl, M.; J. Comb. Chem. 1999, 1, 3. Para uma pesquisa mais atualizada: http: \www.5z.com\divinfo

8. A desconvolução da coleção pode ser realizada por várias estratégias, discutidas em detalhes em vários artigos de revisão (como as referências 1, 2, 5, 9 e 10).

9. Lam, K. S.; Lebl, M.; Krchnak, V.; Chem. Rev. 1997, 97, 411

10. Pirrung, M. C.; Chem. Rev. 1997, 97, 473.

11. Yan, B.; Acc. Chem. Res. 1998, 31, 621.

12. James, I. W.; Tetrahedron 1999, 55, 4855.

13. Hermkens, P. H. H.; Ottenheijm, H. C. J.; Rees, D. C.; Tetrahedron 1996, 52, 4527.

14. Hermkens, P. H. H.; Ottenheijm, H. C. J.; Rees, D. C.; Tetrahedron 1997, 53, 5643.

15. Booth, S.; Hermkens, P. H. H.; Ottenheijm, H. C. J.; Rees, D. C.; Tetrahedron 1998, 54, 15385.

16. Lorsbach, B. A.; Kurth, M. J.; Chem. Rev. 1999, 99, 1549.

17. Gayo, L. M.; Biotechnol. Bioeng. 1998, 61, 95.

18. Suto, M. J.; Gayo, L. M. Palanki, M. S. S.; Sullivan, R.; Tetrahedron 1998, 54, 4141.

19. Kaldor, S. W.; Fritz, J. E.; Tang, J.; McKinney, E. R.; Bioorg. Med. Chem. Lett. 1996, 24, 3041.

20. Kaldor, S. W.; Siegel, M. G.; Fritz, J. E.; Dressman, B. A.; Hahn, P. J.; Tetrahedron Lett. 1996, 37, 7193.

21. Booth, R.J.; Hodges, J. C.; J. Am. Chem. Soc. 1997, $119,4882$.

22. Gravert, D. J.; Janda, K. D.; Chem. Rev. 1997, 97, 489.

23. Molteni, V.; Annunziata, R.; Cinquini, M.; Cozzi, F.; Benaglia, M.; Tetrahedron Lett. 1998, 39, 1257.

24. Fassina, G. Comunicação oral durante o "Workshop on Combinatorial Chemistry and Combinatorial Technologies", Buenos Aires, 7-11 de dezembro de 1998. Universidade de Buenos Aires e International Centre for Science Development and High Technology (ICS/UNIDO).

25. Watson, C.; Angew. Chem. Int. Ed. Engl. 1999, 38, 1903.

26. Carell, T.; Winter, E. A.; Rebek Jr., J.; Angew. Chem. Int. Ed. Engl. 1994, 32, 2061.

27. Powers, D. G.; Casebier, D. S.; Fokas, D.; Ryan, W. J.; Troth, J. R.; Coffen, D. L.; Tetrahedron 1998, 54, 4085.

28. Bunin, B. A.; Plunkett, M. J.; Ellman, J. A.; Method. Enzymol. 1996, 267, 448.

29. Chen, S. Q.; Janda, K. D.;. J. Am. Chem. Soc. 1997, 119,8724 .

30. Hoekstra, W. J.; Maryanoff, B. E.; Andrade-Gordon, P.; Cohen, J. H.; Costanzo, M. J.; Damiano, B. P.; Haertlein, B. J.; Harris, B. D.; Kauffman, J. A.; Keane, P. M.; McComsey, D. F.; Villani Jr, F. J.; Yabut, S. C.; Bioorg. Med. Chem. Lett. 1996, 6, 2371.

31. Salemme, F. R.; Spurlino, J.; Bone, R.; Structure 1997, 5,319 . 
32. Stewart, K. D.; Loren, S.; Frey, L.; Otis, E.; Klinghofer, V.; Hulkower, K. I.; Bioorg. Med. Chem. Lett. 1998, 8, 529.

33. Lew, A.; Chamberlin, A. R.; Bioorg. Med. Chem. Lett. 1999, 9, 3267.

34. Leitão, A.; Montanari, C. A.; Donnici, C. L.; Quim. Nova 2000, 23, 178.

35. Gragg, G. M.; Newmann, D. J.; Snader, K. M.; J. Nat. Prod. 1997, 60, 52.

36. Harvey, A. L.; Trends Pharmacol. Sci. 1999, 20, 196.

37. Fecik, R. A.; Frank, K. E.; Gentry, E.J.; Mitscher, L. A.; Shibata, M.; Pure Appl. Chem. 1999, 71, 559.
38. Nielsen, J.; Lyngso, L. O.; Tetrahedron Lett. 1996, 37, 8439.

39. Meseguer, B.; Alonso-Díaz, D.; Griebenow, N.; Herget, T.; Waldmann, H.; Angew. Chem. Int. Ed. Engl. 1999, 38, 2902.

40. Yang, Z.; He, Y.; Vourloumis, D.; Vallberg, H.; Nicolaou, K. C.; Angew. Chem. Int. Ed. Engl. 1997, 36, 166.

41. Nicolaou, K.C.; Wissinger, N.; Vourloumis, D.; Ohshima, T.; Kim, S.; Pfefferkorn, J.; Xu, Y. J.; Li, T.; J. Am. Chem. Soc. 1998, 120, 10814.

42. Nicolaou, K. C.; Hepworth, D.; King, N. P.; Finlay, M. R. V.; Pure Appl. Chem. 1999, 71, 989. 